\title{
Neutral Diclofenac Causes Remarkable Changes in Phosphatidylcholine Bilayers: Relevance for Gastric Toxicity Mechanisms ${ }^{[S}$
}

\author{
(D) Catarina Pereira-Leite, ${ }^{1,2}$ Sarah K. Jamal, ${ }^{2}$ João P. Almeida, (D)Ana Coutinho, \\ (D) Manuel Prieto, (Dlolanda M. Cuccovia, (1)Cláudia Nunes, and (D) Salette Reis \\ LAQV, REQUIMTE, Departamento de Ciências Químicas, Faculdade de Farmácia, Universidade do Porto, Porto, Portugal \\ (C.P.-L., S.K.J., J.P.A., C.N., S.R.); Departamento de Bioquímica, Instituto de Química, Universidade de São Paulo, São Paulo, Brazil \\ (C.P.-L., I.M.C.); IBB-Institute for Bioengineering and Biosciences, Instituto Superior Técnico (A.C., M.P.) and Departamento de \\ Química e Bioquímica, Faculdade de Ciências (A.C.), Universidade de Lisboa, Lisboa, Portugal
}

Received September 25, 2019; accepted January 24, 2020

\section{ABSTRACT}

The main objective of this study was to clarify the topical mechanisms underlying diclofenac-induced gastric toxicity by considering for the first time both ionization states of this nonsteroidal anti-inflammatory drug. 1,2-Dimyristoyl-snglycero-3-phosphocholine (DMPC) liposomes were the model system chosen to mimic the protective phospholipid layers of the gastric mucosa and to describe the interactions with diclofenac, considering the $\mathrm{pH}$ gradient found in the gastric mucosa $(3<\mathrm{pH}<7.4)$. Complementary experimental techniques were combined to evaluate the drug's affinity for DMPC bilayers, as well as to assess the drug's effects on the structural properties of the phospholipid bilayer. The diclofenac-DMPC interactions were clearly dependent on the drug's ionization state. Neutral diclofenac displayed greater affinity for DMPC bilayers than anionic diclofenac. Moreover, the protonated/ neutral form of the drug induced more pronounced and/or distinct alterations in the structure of the DMPC bilayer than the deprotonated/ionized form, considering similar membrane concentrations. Therefore, neutral diclofenac-induced changes in the structural properties of the external phospholipid layers of the gastric mucosa may constitute an additional toxicity mechanism of this worldwide-used drug, which shall be considered for the development of safer therapeutic strategies.

\section{SIGNIFICANCE STATEMENT}

Neutral or anionic diclofenac exerted distinct alterations in phosphatidylcholine bilayers, which are used in this work as models for the protective phospholipid layers of the gastric mucosa. Remarkable changes were induced by neutral diclofenac in the structural properties of the phospholipid bilayer, suggesting that both ionized and neutral states of nonsteroidal anti-inflammatory drugs must be considered to clarify their mechanisms of toxicity and to ultimately develop safer antiinflammatory drugs.

\section{Introduction}

Diclofenac is one of the most used nonsteroidal antiinflammatory drugs (NSAIDs) worldwide (McGettigan and Henry, 2013). This pharmaceutical is used for the relief of pain and inflammation in chronic musculoskeletal disorders (rheumatoid arthritis, osteoarthritis) and acute painful conditions (renal colic, dysmenorrhea, migraine) (Sweetman, 2011). Diclofenac therapy is associated with an increased risk of

This work was supported by Fundação para a Ciência e a Tecnologia/ Ministério da Ciência, Tecnologia e Ensino Superior (FCT/MCTES) through national funds [Grant UIDB/50006/2020].

${ }^{1}$ Current affiliation: CBIOS - Universidade Lusófona's Research Center for Biosciences and Health Technologies, Lisboa, Portugal

${ }^{2}$ C.P.-L. and S.K.J. contributed equally to this work.

https://doi.org/10.1124/mol.119.118299.

S This article has supplemental material available at molpharm.aspetjournals.org. gastrointestinal (GI), cardiovascular, hepatic, and renal toxicity (Sweetman, 2011; Altman et al., 2015). GI adverse effects are the most frequent in patients taking diclofenac orally, and common reactions include epigastric pain, nausea, vomiting, and diarrhea (Sweetman, 2011).

Diverse GI toxicity mechanisms have been reported for NSAIDs. Prostaglandins (PGs) synthetized by the cyclooxygenase (COX)- 1 pathway are involved in maintaining the protective properties of the GI mucosa by regulating gastric acid secretion, mucus and bicarbonate production, and mucosal blood flow (Wallace, 2008). As the mechanism of action of NSAIDs consists of inhibiting PG production through the COX pathway (Conaghan, 2012), the biosynthesis suppression of COX-1-derived PGs is accepted as a GI toxicity mechanism of NSAIDs (Wallace, 2008). In addition, the topical actions of NSAIDs in the GI tract also seem to contribute to the

ABBREVIATIONS: ANS, 1-anilinonaphthalene-8-sulfonate; AS, (9-anthroyloxy)stearic acid; COX, cyclooxygenase; $d$, distance; DMPC, 1,2dimyristoyl-sn-glycero-3-phosphocholine; $\mathrm{DPH}, 1,6$-diphenyl-1,3,5-hexatriene; $\mathrm{Gl}$, gastrointestinal; $\mathrm{L}_{\alpha}$, fluid phase; $\mathrm{L}_{\beta}$, fluid phase; Log $D_{\mathrm{o} / \mathrm{w}}$, octanol:water log $D$ prediction; LUV, large unilamellar vesicle; NSAID, nonsteroidal anti-inflammatory drug; $\mathrm{P}_{\beta}$, ripple phase; PG, prostaglandin; SAXS, small-angle X-ray scattering; $T_{m}$, main phase temperature; TMA-DPH, 1-(4-trimethylammoniumphenyl)-6-phenyl-1,3,5-hexatriene p-toluenesulfonate; WAXS, wide-angle X-ray scattering. 
occurrence of NSAID-induced GI toxicity. For instance, NSAID trapping and/or NSAID-induced uncoupling of oxidative phosphorylation may cause epithelial cell death in the GI mucosa (Wallace, 2008). In the 1980s, Lichtenberger et al. (1983) and Hills and Lichtenberger (1985) described the protective role of surface-active phospholipids in the gastric mucosa. Diverse studies on NSAID-phospholipid interactions have later demonstrated the ability of NSAIDs to disturb the properties of phospholipid layers, such as their hydrophobicity, fluidity, permeability, and stability (Lichtenberger et al., 2012; Pereira-Leite et al., 2013b). These studies support that the NSAID-induced deleterious actions on membrane and/or external phospholipids of the GI mucosa constitute another key toxicity mechanism of these pharmaceuticals.

In this study, we aim to decipher the effects caused by each ionization state of diclofenac on the phospholipid layers of the gastric mucosa (Fig. 1). This study is of utmost importance for three main reasons: 1) the gastric mucosal barrier presents a $\mathrm{pH}$ gradient from $\mathrm{pH} \sim 2$ in the gastric lumen to $\mathrm{pH} \sim 7$ in the mucosal epithelium (Bahari et al., 1982); 2) diclofenac is an acidic drug $\left[p K_{a}=3.97\right.$ (Ferreira et al., 2005)]; thus, both forms will circulate in the gastric mucosa; and 3) although NSAIDs are recommended to be administrated after meals, when the luminal gastric $\mathrm{pH}$ rises to $2.5-5$ depending on the type of meal (Simonian et al., 2005), both ionized and neutral states of diclofenac may interact with the phospholipid layers, as the $p K_{a}$ of NSAIDs has been reported to increase by $\sim 1$ value in phosphatidylcholine bilayers (de Castro et al., 2001; Pereira-Leite et al., 2018c). In this sense, it is crucial to study the diclofenac interactions with phospholipids at a wider range of $\mathrm{pH}$ values than that reported in the literature (Ferreira et al., 2005; Manrique-Moreno et al., 2009; Suwalsky et al., 2009; Fernandes et al., 2018) to completely decipher its topical mechanism of toxicity in the gastric mucosa.

Therefore, this work describes the interactions of diclofenac with 1,2-dimyristoyl-sn-glycero-3-phosphocholine (DMPC) bilayers at $\mathrm{pH}$ 3.0, 5.0, and 7.4. DMPC liposomes were chosen as model systems because the most abundant phospholipid in the human gastric mucosa is phosphatidylcholine (Nardone et al., 1993). DMPC was also chosen because most glycerophospholipids found in vivo are in the fluid phase (van Meer et al., 2008), as is DMPC at physiologic temperature.

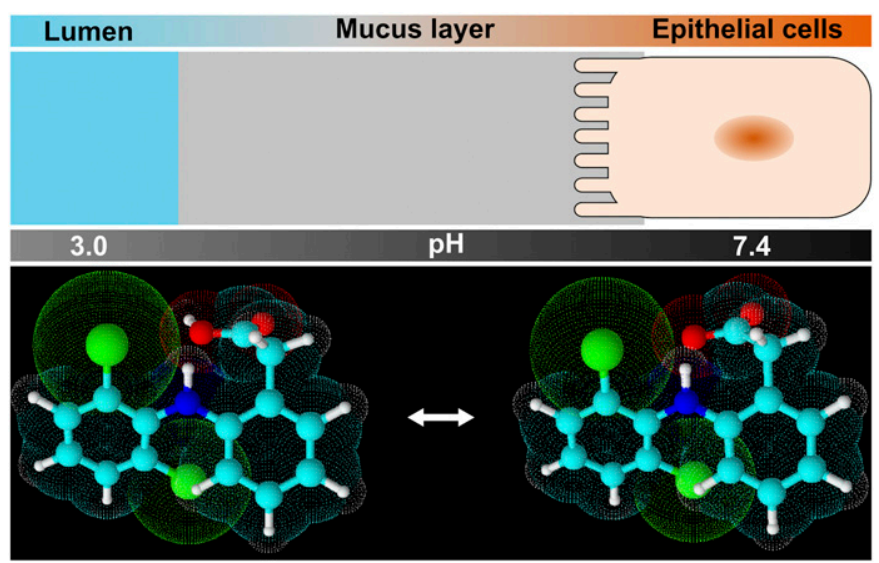

Fig. 1. Schematic representation of the gastric mucosa (upper panel) and 3D chemical structure of diclofenac's ionization states from ACD/ChemSketch 12.01 (lower panel). 3D, three-dimensional.
Moreover, DMPC, a saturated phospholipid, allows the study of ordered and disordered phases simply by varying temperature, enabling the study of drug interactions at diverse physical states of the bilayer. Although diclofenac's maximum plasma concentrations are around $1.7 \mathrm{mg} \mathrm{l}^{-1}$ after an oral intake of $50 \mathrm{mg}$ (Kirchheiner et al., 2003), higher concentrations of diclofenac were tested herein to mimic the gastric content of diclofenac after oral administration and the reported trapping of NSAIDs in the epithelial cells of the gastric mucosa (Wallace, 2008). In this study, the affinity of diclofenac for phosphatidylcholine bilayers was assessed by derivative spectrophotometry and a fluorescence titration technique, whereas the drug's effects on the bilayer structure were evaluated by combining fluorescence anisotropy studies with synchrotron X-ray scattering measurements. Overall, this work is an essential step to comprehensively describe the toxicity mechanisms of diclofenac in the gastric mucosa.

\section{Materials and Methods}

Materials. Diclofenac sodium salt, Trizma base, acetic acid, DMSO, sodium chloride, and Ludox AM-30 colloidal silica were purchased from Sigma-Aldrich (St. Louis, MO). DMPC was obtained from Avanti Polar Lipids, Inc. (Alabaster, AL). The fluorescent probes, 1-(4-trimethylammoniumphenyl)-6-phenyl-1,3,5-hexatriene p-toluenesulfonate (TMA$\mathrm{DPH})$, 1,6-diphenyl-1,3,5-hexatriene (DPH), and 1-anilinonaphthalene-8-sulfonate (ANS), were supplied by Molecular Probes (Invitrogen Corporation, Carlsbad, CA). Formic acid was supplied by Merck \& Co. (Kenilworth, NJ), and chloroform and methanol were supplied by VWR International S.A.S. (Fontenais-sous-Bois, France). All reagents were at least analytical grade and were used with no further purification.

Formate (10 mM, pH 3.0), acetate (10 mM, pH 5.0), and Tris-HCl (10 $\mathrm{mM}, \mathrm{pH}$ 7.4) buffers were prepared by dissolving formic acid, acetic acid, or Trizma base (respectively) in double-deionized water $(\kappa<0.1$ $\mu \mathrm{S} . \mathrm{cm}^{-1}$ ) and adjusting $\mathrm{pH}$ with $\mathrm{NaOH}$ or $\mathrm{HCl}$ solutions $(1 \mathrm{M}) . \mathrm{NaCl}$ $(0.1 \mathrm{M})$ was added to all buffered solutions to adjust the ionic strength.

Liposome Preparation. The lipid film hydration method followed by extrusion was used to prepare large unilamellar vesicles (LUVs) of DMPC, as detailed elsewhere (Pereira-Leite et al., 2018b). A rotary evaporator coupled with $\mathrm{N}_{2}$ stream and water bath at $40^{\circ} \mathrm{C}$ was used to yield lipid films. Lipid films were then hydrated with a buffered solution and extruded using polycarbonate filters (100-nm pore size, Nucleopore Track-Etch Membrane; Whatman) at $40^{\circ} \mathrm{C}$ to produce DMPC LUVs. TMA-DPH-labeled or DPH-labeled LUVs were prepared as previously described, with the additional step of adding the fluorescent probe to the organic solution of DMPC, yielding a probe: lipid molar ratio of 1:300.

Diclofenac was added to DMPC LUVs by incubation or incorporation. The former procedure consisted of dissolving diclofenac in DMSO and adding an aliquot of the drug stock solution to preformed DMPC LUVs, followed by an incubation time of 30 minutes. In the latter procedure, diclofenac was added to the DMPC organic solution before the evaporation process, yielding a drug-lipid film, which was treated as the lipid film to yield multilamellar or unilamellar vesicles. Regardless of the procedure used to add diclofenac, the total amount of DMSO $(2 \%, \mathrm{v} / \mathrm{v})$ was maintained in all experiments. References, i.e., samples without drug, were always tested containing the same percentage of DMSO to consider the basal effects of the solvent, enabling the evaluation of drug effects per se.

Derivative Spectrophotometry. The partition coefficient $\left(K_{p}\right)$ of diclofenac was estimated by derivative spectrophotometry, as described elsewhere (Pereira-Leite et al., 2018b). Samples with increasing concentrations of DMPC $(0-1000 \mu \mathrm{M})$ and a fixed concentration of diclofenac $(40 \mu \mathrm{M})$ were prepared by the incubation procedure. After an incubation period of 30 minutes at $37^{\circ} \mathrm{C}$, the 
samples were transferred to a 96 -well plate, and their absorption spectra $(230-400 \mathrm{~nm})$ were acquired at $37^{\circ} \mathrm{C}$ using a Synergy HT plate reader (BioTek Instruments, Inc.). A $K_{p}$ calculator (Magalhaes et al., 2010) was used to mathematically analyze the experimental data. Dimensionless $\log D\left[\log D=\log \left(K_{p} / V_{\phi}\right)\right]$ was calculated considering a lipid molar volume $\left(V_{\phi}\right)$ of 0.66 l.mol ${ }^{-1}$ (Kucerka et al., 2004).

Fluorescence Titration. A fluorescence titration technique using ANS as fluorophore was performed to assess the diclofenac-membrane binding, in line with previous studies (Pereira-Leite et al., 2013a). Aliquots of an ANS stock solution in DMSO were added to samples with a fixed concentration of DMPC LUVs $(500 \mu \mathrm{M})$ and increasing concentrations of diclofenac $(0-80 \mu \mathrm{M})$ after an incubation period of 30 minutes at $37^{\circ} \mathrm{C}$. The maximum DMSO content in each sample was $2 \%(\mathrm{v} / \mathrm{v})$, in accordance with the other experiments. Spectrofluorimetric data (400-600 nm) were acquired using a Jasco FP-6500 spectrofluorometer by setting the excitation wavelength to $377 \mathrm{~nm}$. ANS adsorption data were mathematically analyzed by fitting eq. 1 to the maximum fluorescence intensity versus ANS concentration plots, from which the binding constant $(K)$, the maximum fluorescence intensity $\left(C_{\max }\right)$, and the binding cooperativity $(b)$ were retrieved.

$$
[A N S]_{B}=C_{\max } \frac{\left(K[A N S]_{\infty}\right)^{b}}{1+\left(K[A N S]_{\infty}\right)^{b}}
$$

\section{Steady-State Fluorescence Anisotropy}

Steady-state fluorescence anisotropy measurements were performed to evaluate the effects of diclofenac on the phase transition behavior of DMPC LUVs and on the membrane fluidity at physiologic temperature, as previously reported (Nunes et al., 2013; Pereira-Leite et al., 2013a). TMA-DPH and DPH were used as fluorescent probes because of their well known and distinct preferential location inside phosphatidylcholine bilayers (Kaiser and London, 1998). Samples with a fixed concentration of labeled DMPC LUVs $(500 \mu \mathrm{M})$ and increasing concentrations of diclofenac $(0-80 \mu \mathrm{M})$ were prepared and incubated at $37^{\circ} \mathrm{C}$ for 30 minutes. Steady-state fluorescence anisotropy data were recorded using a Jasco FP-6500 spectrofluorometer coupled with Jasco ADP 303T temperature controller, enabling the acquisition of temperature-dependent scans. The excitation/emission wavelengths were set to $359 / 429$ and $357 / 429 \mathrm{~nm}$ for TMA-DPH and $\mathrm{DPH}$, respectively. Temperature-dependent $\left(10-40^{\circ} \mathrm{C}\right.$, data acquisition interval $2^{\circ} \mathrm{C}$ ) and temperature-fixed $\left(37^{\circ} \mathrm{C}\right)$ experiments were performed to evaluate the lipid phase transition and the membrane fluidity at physiologic temperature, respectively.

Temperature-dependent results were mathematically treated by plotting the fluorescence anisotropy $\left(r_{s}\right)$ versus temperature $(T)$ and by fitting eq. 2 to the experimental data to determine the temperature $\left(T_{m}\right)$ and the cooperativity $(B)$ of the main phase transition of DMPC in the absence and presence of drug (Grancelli et al., 2002).

$$
r_{s}=r_{S 1}+p_{1} T+\frac{r_{s 2}-r_{s 1}+p_{2} T-p_{1} T}{1+10^{B\left(1 / T-1 / T_{m}\right)}}
$$

In this equation, $p_{1}$ and $p_{2}$ are the slopes of linear fits to the data before and after the phase transition region (respectively), and $r_{s 1}$ and $r_{s 2}$ are the corresponding $y$-intercepts.

Temperature-fixed data were considered to evaluate the drug's effect on the bilayer fluidity. According to the wobbling-in-cone model, the steady-state anisotropy values $(\langle r\rangle)$ of embedded probes depend on the fluorophore lifetime $(\tau)$, as well as on the rotational correlation time $(\theta)$ and the limiting anisotropy $\left(r_{\infty}\right)$, as described by eq. 3 (Lakowicz, 2006).

$$
r=\frac{r_{0}}{1+\frac{\tau}{\theta}}+r_{\infty}
$$

The decrease of the probe $\tau$ values upon membrane partitioning of the drug can be either due to collisional quenching or higher hydration of the membrane interface; either way, this effect can cause a subsequent increase of $\langle r\rangle$ values, which is unrelated to alterations in bilayer fluidity. Thus, corrected steady-state anisotropy values $\left(\langle r\rangle_{c o r}\right)$ were calculated (eq. 4) to eliminate the contribution of the variations of fluorophore lifetime, enabling the estimation of the drug's effects on the bilayer fluidity.

$$
r_{\text {cor }}=\frac{\left(\theta+\tau_{\text {exp }}\right)}{\left(\theta+\tau_{0}\right)} \times r_{\text {exp }}
$$

In eq. 4 , the experimental steady-state anisotropy values $\left(\langle r\rangle_{\text {exp }}\right)$ were corrected considering the probes' lifetime in the absence and presence of drug $\left(\langle\tau\rangle_{0}\right.$ and $\langle\tau\rangle_{\text {exp }}$, respectively) and the rotational correlation time of the probes $(\theta)$. All parameters used for this correction were experimentally obtained as described in the Supplemental Methods.

\section{Synchrotron X-Ray Scattering}

Synchrotron X-ray scattering measurements were performed to assess the effect of diclofenac on the structure of the DMPC bilayer, as previously described (Pereira-Leite et al., 2018c). Stacked lipid bilayers of DMPC containing various molar ratios of diclofenac (0-0.16) were prepared from multilamellar vesicles produced by the incorporation method. After a storage period of at least 1 week at $4^{\circ} \mathrm{C}$ in transparent capillaries, the samples were analyzed in the noncrystalline diffraction beamline of ALBA synchrotron (Cerdanyola del Vallès, Spain), yielding the small-angle and wide-angle X-ray scattering (SAXS and WAXS, respectively) patterns of DMPC. The patterns obtained at 10 and $37^{\circ} \mathrm{C}$ were mathematically analyzed using OriginPro 8.5 software by fitting the Lorentzian function to the peaks obtained to retrieve the maximum position $(q)$ and the full width of half maximum (fwhm) of each peak. These values were then used to calculate the longand short-range distances $(d=2 \pi / q)$, from SAXS and WAXS data (respectively), and the correlation lengths $\left(\xi=4 \pi^{2} / f w h m\right)$.

\section{Results}

Diclofenac's Affinity for Phosphatidylcholine Bilayers. The affinity of diclofenac for DMPC bilayers was first evaluated by the determination of the partition coefficient at $37^{\circ} \mathrm{C}$ and $\mathrm{pH} 3.0,5.0$, and 7.4 by UV-Vis derivative spectrophotometry (Table 1 ). The obtained $\log D$ values were $\mathrm{pH}$-dependent and higher for lower $\mathrm{pH}$ values, suggesting a distinct affinity of each ionization state of the drug for the DMPC bilayer.

In liposome-water systems, the acid-base equilibria of acidic drugs (Supplemental Scheme 1), such as diclofenac, take into account the partition coefficient of each ionization state, as well as the $p K_{a}$ in the bulk phase $\left(K_{a}\right)$ and in the lipid phase $\left(K_{m}\right)$ (Avdeef, 2001). From the determined $\log D$ values and the reported $p K_{a}$ of diclofenac $(3.97 \pm 0.04)$ in the aqueous medium (Ferreira et al., 2005), the apparent $p K_{m}$ of diclofenac was calculated, as previously reported (de Castro et al., 2001;

\section{TABLE 1}

$\log D$ of diclofenac at $37^{\circ} \mathrm{C}$ as a function of medium $\mathrm{pH}$ determined by UV-Vis derivative spectrophotometry

$\log D$ values are presented as means \pm S.D. of at least three independent assays. Log $D_{\mathrm{o} / \mathrm{w}}$ values from MarvinSketch calculator (ChemAxon) are also presented.

\begin{tabular}{ccc}
\hline $\mathrm{pH}$ & $\log D$ & $\log D_{\mathrm{o} / \mathrm{w}}$ \\
\hline 3.0 & $4.13 \pm 0.07$ & 4.22 \\
5.0 & $3.74 \pm 0.07$ & 3.21 \\
7.4 & $3.49 \pm 0.03$ & 1.10 \\
\hline
\end{tabular}

$\log D_{\mathrm{o} / \mathrm{w}}$, octanol:water $\log D$ prediction. 
Pereira-Leite et al., 2018c), and was found to be $4.7 \pm 0.3$. This means that the protonated/neutral form of diclofenac is predominant in the DMPC bilayer at $\mathrm{pH} 3.0$, whereas the anionic form prevails at $\mathrm{pH}$ 7.4. In this sense, and considering the experimental $\log D$ values, neutral diclofenac has higher affinity for the DMPC bilayer than anionic diclofenac.

The mathematical predictions of $\log D$, considering the octanol-water system, were markedly different from the experimental $\log D$ values at $\mathrm{pH} 7.4$ and 5.0 (Table 1 ), whereas no pronounced differences were found at $\mathrm{pH}$ 3.0. The higher experimental values suggest that nonhydrophobic interactions drive the partitioning of anionic diclofenac with the DMPC bilayer because the octanol-water system only takes into account hydrophobic interactions (Magalhaes et al., 2010). In contrast, nonpolar interactions may be on the basis of neutral diclofenac interactions with DMPC bilayers because the predicted and experimental values were similar. To test these hypotheses, diclofenac-DMPC binding studies were performed using a titration technique with an anionic fluorophore, i.e., ANS.

The ANS-DMPC binding parameters retrieved from the ANS binding isotherms (Supplemental Fig. 1) are presented in Table 2. No pronounced alterations in the binding parameters were observed upon diclofenac addition beyond the concentration-dependent decrease of $C_{\max }$ at $\mathrm{pH} 3.0$ and 5.0. Because ANS is an anionic probe reported to be located at the headgroup region of phosphatidylcholine bilayers (Jendrasiak and Estep, 1977), it was initially expected that anionic diclofenac would greatly compete for the ANS binding sites. Nevertheless, neutral diclofenac seems to be the drug's ionization state competing with ANS to the DMPC binding sites because $C_{\max }$ only decreased at $\mathrm{pH}$ values in which the neutral species is present. These results may be justified by the membrane concentration of diclofenac at each $\mathrm{pH}$ value and/or by the type of interactions established between each ionization state of diclofenac and DMPC.

The membrane concentration of diclofenac at each $\mathrm{pH}$ value was calculated from the experimentally determined partition coefficient $\left(K_{p}\right)$ values using eq. 5 , as previously reported (Lakowicz, 2006; Nunes et al., 2011):

$$
[\text { Drug }]_{m}=\frac{K_{p}[\text { Drug }]}{1+\left(K_{p}-1\right)\left(V_{\phi}[L]\right)}
$$

[Drug] stands for the total molar concentration of diclofenac, $[L]$ is the DMPC molar concentration, and $V_{\phi}$ is the DMPC molar volume. It is noteworthy that $0.1 \mathrm{M}$ of diclofenac at $\mathrm{pH}$
3.0 caused a decrease of $C_{\max }$, whereas no changes were observed in the binding parameters with $0.12 \mathrm{M}$ of diclofenac at $\mathrm{pH} 7.4$ (Table 2). This result indicates that the ANSdiclofenac competition is, in fact, more significant at $\mathrm{pH}$ values in which the drug is protonated/neutral. This evidence suggests that polar interactions may also drive the partitioning of neutral diclofenac with the DMPC bilayer. Among polar interactions, ANS may interact with DMPC by H-bonding between the ANS's NH group and the DMPC phosphate group and by ionic interactions between the ANS's sulfonate group and the DMPC choline group. Thus, the competition for the $\mathrm{H}$-bonding seems to be more relevant than that for the ionic interaction, as anionic diclofenac did not disturb the ANS binding to the DMPC bilayer, whereas neutral diclofenac did. Indeed, the protonated/neutral form of the drug may also establish carboxylic acid-phosphate H-bonding, hampering the interaction between ANS and DMPC. The higher competition observed with neutral diclofenac may also be supported by the hydrophilic-lipophilic balance of the two ionization states of diclofenac: 4.67 for neutral diclofenac and 21.92 for anionic diclofenac, according to MarvinSketch calculator (Chemaxon). These values indicate that a higher energetic barrier may hinder the removal of neutral diclofenac from the bilayer into the bulk phase to accommodate ANS, whereas anionic diclofenac may be easily dislocated to the aqueous phase because of its more hydrophilic nature.

Altogether, the experimental data showed that neutral diclofenac has a higher affinity for the DMPC bilayer and that both ionization states of diclofenac may interact with the headgroup region of the DMPC bilayer. Ionic bonding with the choline moiety of DMPC may drive the anionic diclofenac partitioning, whereas H-bonding with the phosphate moiety of DMPC may be on the basis of neutral diclofenac incorporation.

Diclofenac's Effects on the Structural Properties of Phosphatidylcholine Bilayers. The effects of diclofenac on the main phase transition of DMPC LUVs were evaluated at $\mathrm{pH} 3.0,5.0$, and 7.4 by temperature-dependent steady-state fluorescence anisotropy measurements. Two distinct fluorescent probes (TMA-DPH and DPH) were embedded in the DMPC LUVs to retrieve fluorescence anisotropy data. As an example, the sigmoid curves obtained with TMA-DPHlabeled and DPH-labeled DMPC LUVs at $\mathrm{pH} 3.0$ are presented in Fig. 2. The results obtained at $\mathrm{pH} 5.0$ and 7.4 are available in Supplemental Fig. 2. By fitting eq. 2 to the experimental data, the temperature $\left(T_{m}\right)$ and the cooperativity $(B)$ of the main phase transition of DMPC in the absence and presence of diclofenac were estimated (Table 3 ).

TABLE 2

ANS-DMPC binding parameters—namely, binding constant $(K)$, maximum fluorescence intensity $\left(C_{\max }\right)$, and binding cooperativity $(b)$ - upon addition of diclofenac at $37^{\circ} \mathrm{C}$ as a function of $\mathrm{pH}$ value

The values of the binding parameters are presented as the means \pm S.D. of two independent assays. The total molar concentration of diclofenac added ([Drug]) and the corresponding membrane concentration ([Drug $]_{\mathrm{m}}$ ) are also presented.

\begin{tabular}{|c|c|c|c|c|c|}
\hline $\mathrm{pH}$ & {$\left[\right.$ Drug] $\left(\times 10^{-4} \mathrm{M}\right)$} & {$[\text { Drug }]_{\mathrm{m}}\left(\times 10^{-2} \mathrm{M}\right)$} & $K$ & $b$ & $C_{\max }$ \\
\hline \multirow[t]{3}{*}{3.0} & 0 & 0 & $0.08 \pm 0.01$ & $1.40 \pm 0.06$ & $1049 \pm 86$ \\
\hline & 4 & 10 & $0.076 \pm 0.004$ & $1.34 \pm 0.03$ & $880 \pm 59$ \\
\hline & 8 & 20 & $0.074 \pm 0.006$ & $1.36 \pm 0.01$ & $747 \pm 63$ \\
\hline \multirow{3}{*}{5.0} & 0 & 0 & $0.069 \pm 0.001$ & $1.29 \pm 0.05$ & $1005 \pm 50$ \\
\hline & 4 & 8 & $0.069 \pm 0.003$ & $1.25 \pm 0.02$ & $916 \pm 34$ \\
\hline & 8 & 16 & $0.067 \pm 0.001$ & $1.27 \pm 0.05$ & $857 \pm 59$ \\
\hline \multirow[t]{3}{*}{7.4} & 0 & 0 & $0.073 \pm 0.001$ & $1.33 \pm 0.01$ & $936 \pm 43$ \\
\hline & 4 & 6 & $0.068 \pm 0.003$ & $1.25 \pm 0.01$ & $976 \pm 6$ \\
\hline & 8 & 12 & $0.074 \pm 0.002$ & $1.33 \pm 0.01$ & $938 \pm 17$ \\
\hline
\end{tabular}


The main phase transition temperature of pure DMPC LUVs was consistent with the literature at all $\mathrm{pH}$ values (Koynova and Caffrey, 1998), irrespective of the embedded probe. It is noteworthy that $T_{m}$ of plain DMPC was slightly superior at $\mathrm{pH} 3.0$, as previously reported (Koynova and Caffrey, 1998), because of the partial protonation of the DMPC's phosphate group ( $p K_{a}=1.85$ according to the MarvinSketch calculator from ChemAxon), which enables the additional formation of $\mathrm{H}$-bonds between lipid molecules.

Diclofenac induced a concentration- and $\mathrm{pH}$-dependent decrease of $T_{m}$ and $B$ of the DMPC bilayer. By comparing similar membrane concentrations of diclofenac at $\mathrm{pH} 3.0(0.1$ $\mathrm{M})$ and $\mathrm{pH} 7.4(0.12 \mathrm{M})$, the experimental data revealed that neutral diclofenac has a more pronounced effect on both parameters than anionic diclofenac. These differential effects of diclofenac according to its state may reflect distinct membrane locations of the protonated and deprotonated forms. Because the results obtained with both fluorescent probes were similar, it is plausible that both forms of diclofenac locate between the headgroup region and the first carbons of the DMPC acyl chains. Noticeably, the neutral diclofenac ( $\mathrm{pH}$ 3.0) influenced to a greater extent the cooperativity of the phase transition than anionic diclofenac $(\mathrm{pH} 7.4)$. This result suggests that neutral diclofenac is closer to the more ordered region of the acyl chains (C1-C8) than the anionic form (Jain and $\mathrm{Wu}, 1977$ ). These data are in line with our hypothesis concerning the putative chemical interactions formed between diclofenac and DMPC, suggesting once again that anionic diclofenac preferentially locates in a shallower region of the bilayer.

The effect of diclofenac on the fluidity of the DMPC bilayer in the fluid phase $\left(37^{\circ} \mathrm{C}\right)$ was also evaluated by steady-state fluorescence anisotropy measurements using TMA-DPH and DPH as fluorescent probes (Supplemental Fig. 3). These experiments do not directly retrieve the drug's effect on membrane fluidity, as changes in the probes' lifetime due to drug incorporation may also lead to alterations in the steadystate anisotropy $\left(\langle r\rangle_{\text {exp }}\right)$ values (Lakowicz, 2006). In this sense, time-resolved fluorescence intensity and anisotropy measurements (Supplemental Fig. 5; Supplemental Table 1) were also performed to correct the $\langle r\rangle_{\text {exp }}$ values according to eq. 4 to

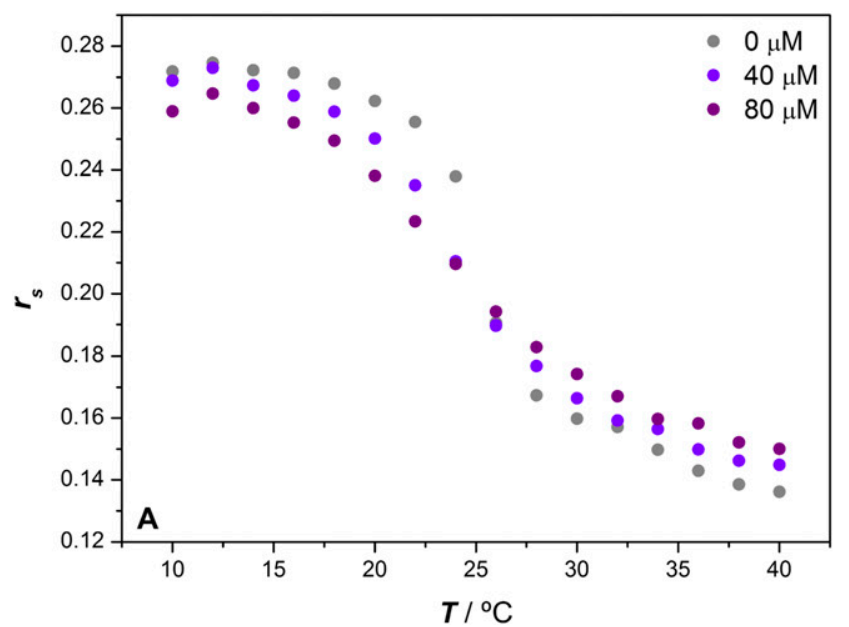

ultimately estimate the effect of diclofenac on the fluidity of the DMPC bilayer.

The obtained values of $\langle r\rangle_{c o r}$ in the absence of drug (Fig. 3) confirm that TMA-DPH and DPH are located in different regions of the bilayer because $\langle r\rangle_{c o r}$ obtained for TMA-DPH is higher than that obtained for DPH. These data are in line with the order gradient reported for the acyl chains of phosphatidylcholine bilayers (Venable et al., 2015), which decreases from the surface to the bilayer center, and corroborate the shallower location of TMA-DPH in the DMPC bilayer in comparison with DPH, as previously reported (Kaiser and London, 1998).

Upon diclofenac's incorporation, no pronounced alterations were observed in the $\langle r\rangle_{c o r}$ values of TMA-DPH (Fig. 3), suggesting that diclofenac does not cause remarkable changes in the fluidity of the shallower region of the DMPC bilayer, irrespective of the drug's ionization state. Considering the bilayer region probed by $\mathrm{DPH}$, a concentration-dependent increase of $\langle r\rangle_{c o r}$ was induced by diclofenac at $\mathrm{pH} 3.0$, but no alterations were found at the other $\mathrm{pH}$ values (Fig. 3). It is noteworthy that the effect of diclofenac on $\langle r\rangle_{c o r}$ depends on the membrane concentration of neutral diclofenac, with a pronounced increase from $0.1 \mathrm{M}$ (Supplemental Fig. 4). This result suggests that neutral diclofenac induces the reduction of the bilayer fluidity of the inner region of the DMPC bilayer, supporting once again that the protonated/neutral form of the drug locates deeper inside the bilayer. By inserting in the inner region of the bilayer, neutral diclofenac may function as a steric restriction, reducing the number of gauche isomers in the DMPC acyl chains, leading to more rigid bilayers.

To further evaluate the influence of diclofenac on the structural properties of the DMPC bilayer, synchrotron X-ray scattering measurements were also performed at 10 and $37^{\circ} \mathrm{C}$. Small-angle (SAXS) and wide-angle (WAXS) data were retrieved to obtain information about the long-range and the short-range organization of the DMPC bilayer, respectively. As an example, the SAXS and WAXS patterns of the DMPC bilayer with increasing diclofenac:lipid molar ratios at pH 7.4 are presented in Fig. 4. From the SAXS and WAXS patterns, the long- and short-range distances $(d)$ were calculated (respectively), giving information about the bilayer thickness and the lipid packing, respectively. Moreover, the

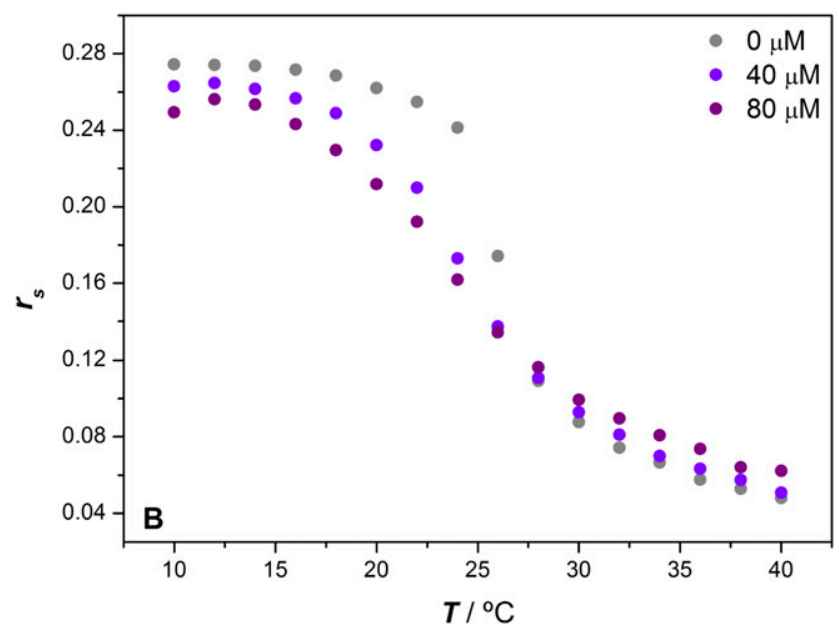

Fig. 2. Temperature-dependent steady-state fluorescence anisotropy of TMA-DPH-labeled DMPC LUVs (A) or DPH-labeled DMPC LUVs (B) in the presence of variable diclofenac concentrations $(0,40$, or $80 \mu \mathrm{M})$ at $\mathrm{pH} 3.0 . r_{s}$, steady-state fluorescence anisotropy; $T$, temperature. 
TABLE 3

Main phase transition temperature $\left(T_{\mathrm{m}}\right)$ and cooperativity $(B)$ of DMPC LUVs as a function of embedded fluorescent probe, medium pH, and diclofenac concentration

The displayed values are the mean \pm S.D. of at least two independent assays. The membrane concentration of diclofenac ([Drug $]_{\mathrm{m}}$ ), calculated from eq. 5 , is also presented.

\begin{tabular}{|c|c|c|c|c|c|c|}
\hline \multirow{2}{*}{$\mathrm{pH}$} & \multirow{2}{*}{ [Drug] $\left(\times 10^{-4} \mathrm{M}\right)$} & \multirow{2}{*}[\text{Drug}]{$_{\mathrm{m}}\left(\times 10^{-2} \mathrm{M}\right)$} & \multicolumn{2}{|c|}{ TMA-DPH } & \multicolumn{2}{|c|}{ DPH } \\
\hline & & & $T_{m} /{ }^{\circ} \mathrm{C}$ & $B$ & $T_{m} /{ }^{\circ} \mathrm{C}$ & $B$ \\
\hline \multirow[t]{3}{*}{3.0} & 0 & 0 & $25.3 \pm 0.5$ & $265 \pm 43$ & $26.0 \pm 0.1$ & $288 \pm 5$ \\
\hline & 4 & 10 & $23.4 \pm 0.1$ & $91 \pm 1$ & $24.7 \pm 0.1$ & $104 \pm 7$ \\
\hline & 8 & 20 & $23.9 \pm 0.1$ & $80 \pm 7$ & $24.9 \pm 0.8$ & $71 \pm 3$ \\
\hline \multirow[t]{3}{*}{5.0} & 0 & 0 & $24.7 \pm 0.1$ & $295 \pm 34$ & $24.7 \pm 0.1$ & $317 \pm 39$ \\
\hline & 4 & 8 & $23.6 \pm 0.3$ & $132 \pm 8$ & $23.7 \pm 0.2$ & $147 \pm 16$ \\
\hline & 8 & 16 & $23.1 \pm 0.3$ & $86 \pm 2$ & $23.2 \pm 0.1$ & $91 \pm 5$ \\
\hline \multirow[t]{3}{*}{7.4} & 0 & 0 & $24.7 \pm 0.1$ & $358 \pm 15$ & $24.9 \pm 0.1$ & $389 \pm 16$ \\
\hline & 4 & 6 & $24.5 \pm 0.1$ & $284 \pm 34$ & $24.8 \pm 0.1$ & $321 \pm 32$ \\
\hline & 8 & 12 & $24.1 \pm 0.1$ & $223 \pm 22$ & $24.5 \pm 0.1$ & $273 \pm 17$ \\
\hline
\end{tabular}

corresponding correlation lengths $(\xi)$ were also determined to evaluate the homogeneity of the long- and short-range distances throughout the DMPC bilayers. Long-range $d$ and $\xi$ values are presented in Table 4 , whereas short-range values are displayed in Supplemental Table 2.

The bilayer thickness of the DMPC bilayer was evaluated at $10^{\circ} \mathrm{C}$ by SAXS measurements. Although the bilayer was expected to be in the gel $\left(\mathrm{L}_{\beta},\right)$ phase at $10^{\circ} \mathrm{C}$, the obtained SAXS patterns of plain DMPC was typical of the ripple $\left(\mathrm{P}_{\beta}\right)$ phase by presenting two first-order and second-order Bragg peaks (Fig. 4), as previously reported (Pereira-Leite et al., 2018c). The calculated long-range distances are in line with the bilayer thicknesses reported for the gel-like domains $\left(d_{1}\right.$ values) and the fluid-like domains ( $d_{2}$ values) of the DMPC's $\mathrm{P}_{\beta}$, phase (Eisenblatter and Winter, 2006; Pereira-Leite et al., 2018 c). It is noteworthy that the partial protonation of the phosphate group of DMPC at $\mathrm{pH} 3.0$ caused an increase of about $4 \AA$ of the bilayer thickness of the fluid-like domains, probably because of the molecular rearrangement of the DMPC headgroup. The incorporation of diclofenac in the DMPC bilayer caused two main alterations: 1) a pronounced increase of the bilayer thickness of both gel-like and fluid-like domains ( $d_{1}$ and $d_{2}$ values) of the DMPC bilayer and 2 ) the destabilization of the DMPC's stacked bilayers, as the typical broad peak of phospholipid vesicles $\left(0.5-2.5 \mathrm{~nm}^{-1}\right)$ was observed for the highest drug:lipid ratio tested at both $\mathrm{pH}$ 5.0 and 7.4, hampering the determination of the long-range distances. It is worth mentioning that a partial disruption of the stacked bilayers was also observed for the intermediate drug:lipid ratio tested at $\mathrm{pH} 5.0$ and 7.4, whereas it was not observed for the same drug:lipid ratio at $\mathrm{pH} 3.0$ (Supplemental Fig. 6). Indeed, the presence of anionic diclofenac at pH 5.0 and 7.4 may hinder the formation of stacked bilayers because of electrostatic repulsion caused by the high superficial density of negative charges.

The diclofenac-induced increase of the bilayer thickness of the DMPC's $\mathrm{P}_{\beta}$, phase may be due to 1 ) an increase of the water layer between phospholipid bilayers and/or 2) a decrease of the tilt angle of DMPC's acyl chains. The latter justification was experimentally addressed by WAXS experiments. As it can be observed in Fig. 4, diclofenac caused the transition from an asymmetric Bragg peak to a symmetric one in WAXS patterns at all $\mathrm{pH}$ values (Supplemental Table 2), indicating that the tilted orthorhombic lipid packing was changed for the untilted hexagonal lipid packing. However, the full straightening of the DMPC molecules would only result in a bilayer thickness increment of $\sim 2.8 \AA$, considering the tilt angle of the DMPC's $\mathrm{P}_{\beta}$, phase $\left(18.4^{\circ}\right)$ (Akabori and Nagle, 2015) and the DMPC

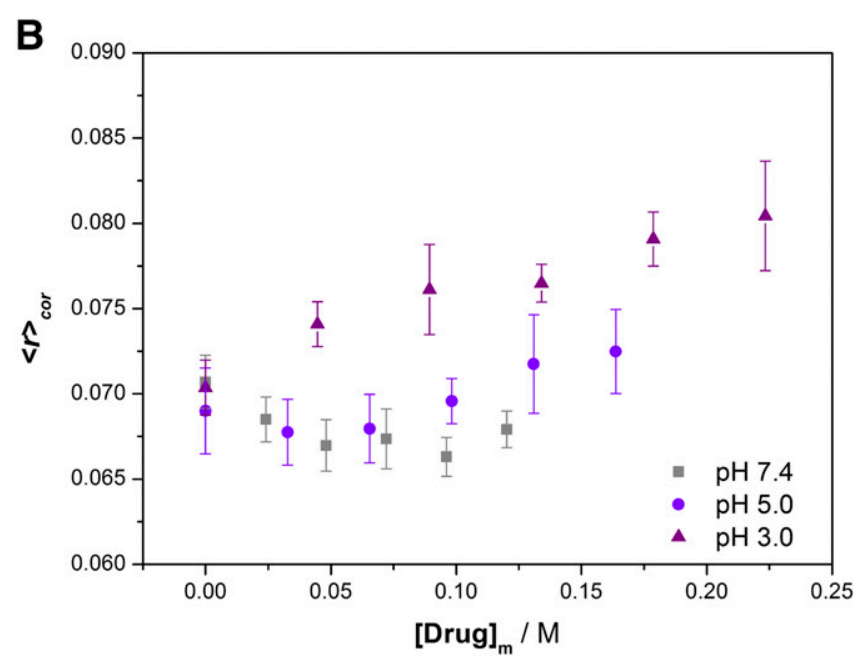

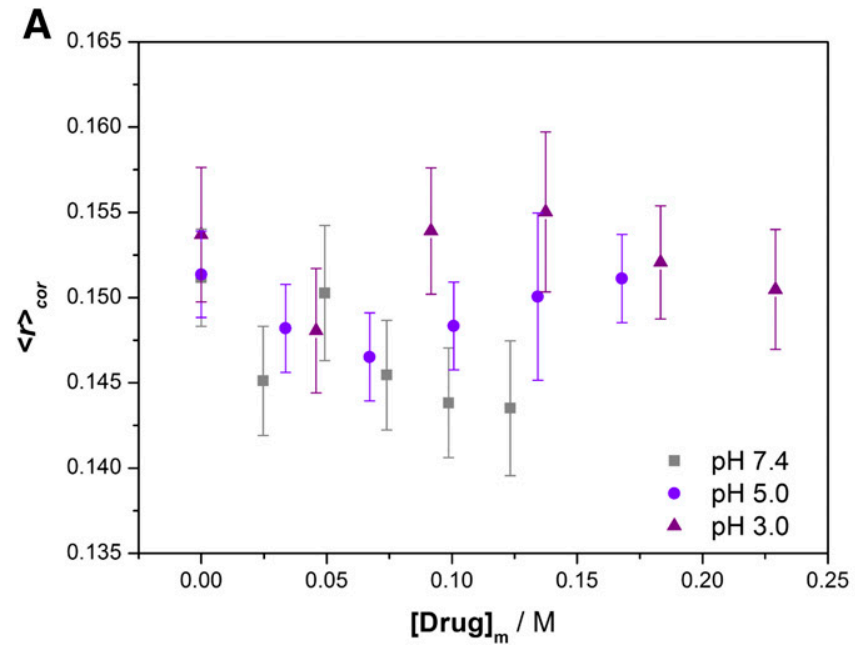

Fig. 3. Corrected steady-state anisotropy values $\left(\langle r\rangle_{c o r}\right)$ of TMA-DPH (A) and DPH (B) embedded in DMPC LUVs at $37^{\circ} \mathrm{C}$ as a function of the membrane concentration of diclofenac $\left([\mathrm{Drug}]_{\mathrm{m}}\right)$ and medium $\mathrm{pH}$. Data are presented as means \pm S.D. of three independent assays, considering the propagation of uncertainty as $\langle r\rangle_{c o r}$ is a calculated parameter obtained from three experimental values. 
SAXS $10^{\circ} \mathrm{C}$

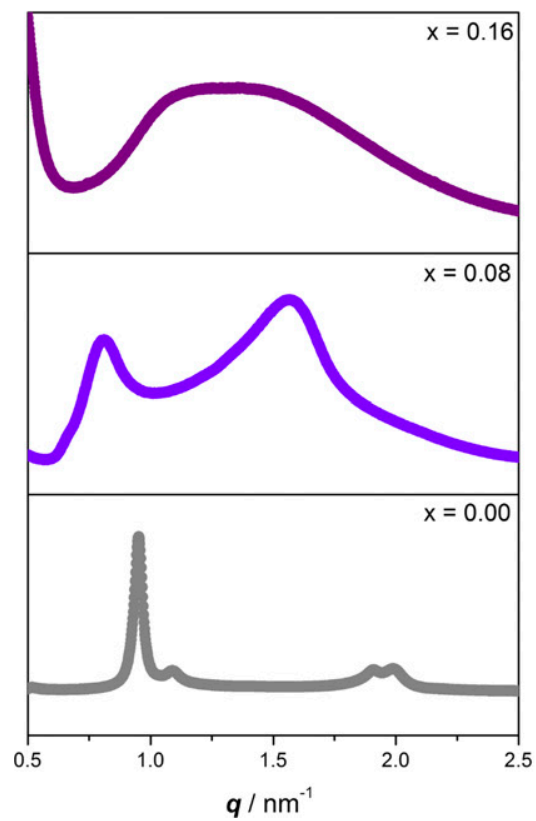

WAXS $10^{\circ} \mathrm{C}$

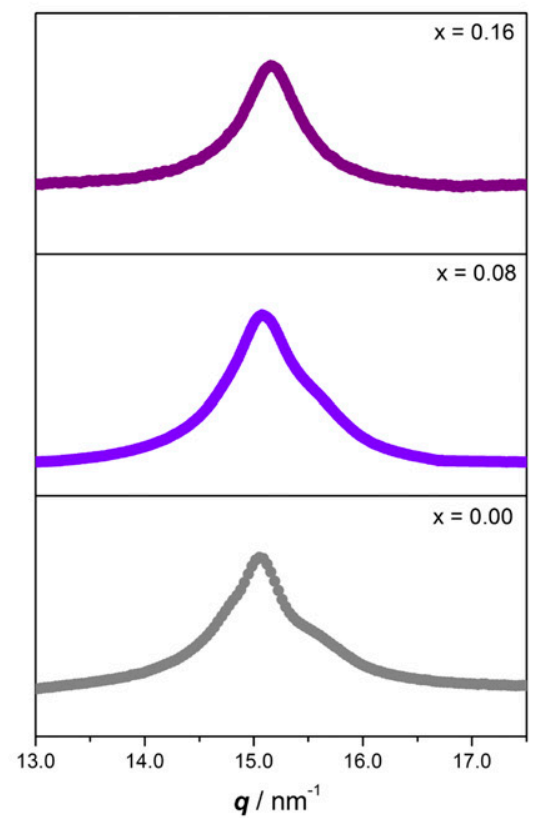

SAXS $37^{\circ} \mathrm{C}$

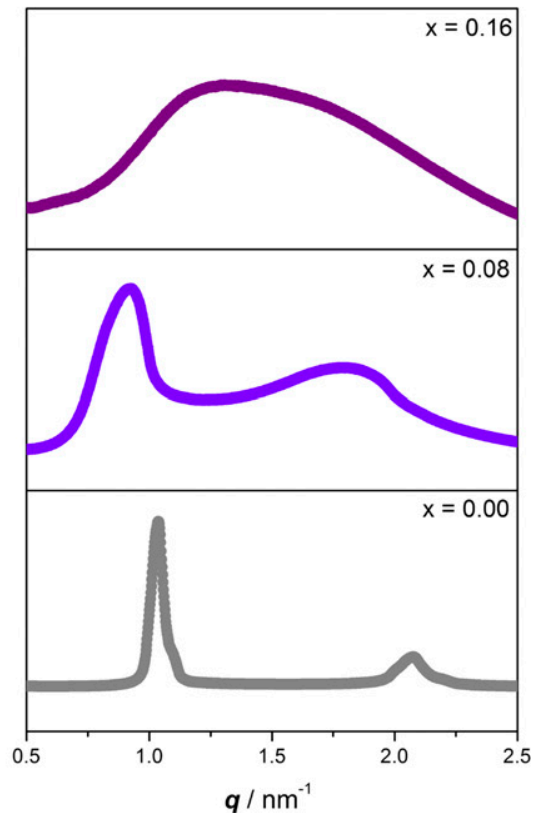

Fig. 4. SAXS and WAXS patterns of DMPC bilayers at $\mathrm{pH} 7.4$ as a function of diclofenac:lipid molar ratio (x) at 10 and $37^{\circ} \mathrm{C} . q$, amplitude of the scattering vector.

length as $\sim 27 \AA$. In this sense, the hydration layer between the DMPC bilayers must also be augmented as the long-range $d$ values increased at least $7 \AA$ upon diclofenac incorporation. Notably, a superior increase of long-range $d$ values was observed at $\mathrm{pH} 7.4$ and 5.0, probably to avoid interbilayer electrostatic repulsion due to the high superficial charge density caused by anionic diclofenac incorporation.

At $37^{\circ} \mathrm{C}$, the plain DMPC bilayers were mainly in the fluid $\left(\mathrm{L}_{\alpha}\right)$ phase, as expected, but a small shoulder was still visible in the SAXS patterns, suggesting that fluid-like domains of the $\mathrm{P}_{\beta}$, phase were still present (Fig. 4; Table 4). In line with the data at $10^{\circ} \mathrm{C}$, the long-range distances of plain DMPC at $\mathrm{pH}$ 3.0 were higher than that at the other $\mathrm{pH}$ values, which was in line with the literature (Petrache et al., 1998; Pereira-Leite et al., 2018c), because of the presence of protonated DMPC molecules. The incorporation of diclofenac in the DMPC bilayers caused an increase of the long-range $d$ values, as well as the disruption of the DMPC stacked bilayers for the higher diclofenac:lipid molar ratio, in agreement with the data at $10^{\circ}$ C. The increase of long-range $d$ values in fluid phases may be related to an increase of the water layers between stacked bilayers and/or to an increase of the trans:gauche ratio due to steric constraints. Considering the middle drug:lipid ratio tested, the long-range $d$ values pronouncedly increased at $\mathrm{pH}$ 7.4 and 5.0, whereas a smaller variation was observed at $\mathrm{pH}$ 3.0. This result may be once again related to the charge

\section{TABLE 4}

Long-range distances $(d)$ and correlation lengths $(\xi)$ calculated from the first-order peak(s) of the SAXS patterns of DMPC bilayers as a function of temperature, $\mathrm{pH}$, and drug:lipid molar ratio $(\mathrm{x})$

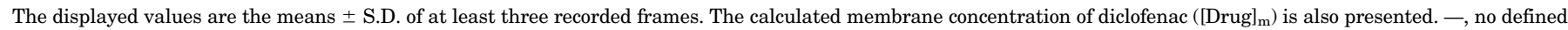
Bragg peaks were observed,

\begin{tabular}{|c|c|c|c|c|c|c|c|}
\hline $\mathrm{T} /{ }^{\circ} \mathrm{C}$ & $\mathrm{pH}$ & $\mathrm{x}$ & {$[\text { Drug }]_{\mathrm{m}} / \mathrm{M}$} & $d_{1} / \AA$ & $\xi_{1} / \AA ̊$ & $d_{2} / \AA$ & $\xi_{2} / \AA ̊$ \\
\hline \multirow[t]{9}{*}{10} & \multirow[t]{3}{*}{3.0} & 0.00 & 0.00 & $65.5 \pm 0.2$ & $8208 \pm 299$ & $61.3 \pm 0.1$ & $7796 \pm 77$ \\
\hline & & 0.08 & 0.12 & $72.6 \pm 0.2$ & $7916 \pm 222$ & $69.6 \pm 0.2$ & $5828 \pm 176$ \\
\hline & & 0.16 & 0.24 & $72.9 \pm 0.4$ & $6902 \pm 997$ & $68.7 \pm 0.6$ & $558 \pm 113$ \\
\hline & \multirow[t]{3}{*}{5.0} & 0.00 & 0.00 & $66.8 \pm 0.1$ & $7035 \pm 30$ & $57.0 \pm 0.1$ & $3111 \pm 135$ \\
\hline & & 0.08 & 0.12 & $84.1 \pm 0.2$ & $5646 \pm 307$ & $76.4 \pm 0.1$ & $3250 \pm 118$ \\
\hline & & 0.16 & 0.24 & - & - & - & - \\
\hline & \multirow[t]{3}{*}{7.4} & 0.00 & 0.00 & $66.1 \pm 0.1$ & $9914 \pm 31$ & $57.4 \pm 0.1$ & $4557 \pm 45$ \\
\hline & & 0.08 & 0.12 & $92 \pm 7$ & $1588 \pm 310$ & $79 \pm 3$ & $2120 \pm 303$ \\
\hline & & 0.16 & 0.24 & - & - & - & - \\
\hline \multirow[t]{9}{*}{37} & \multirow[t]{3}{*}{3.0} & 0.00 & 0.00 & $65.5 \pm 0.3$ & $6503 \pm 328$ & $59.0 \pm 0.6$ & $1596 \pm 194$ \\
\hline & & 0.08 & 0.12 & $64.9 \pm 0.1$ & $11,359 \pm 1734$ & $63.4 \pm 0.1$ & $9804 \pm 428$ \\
\hline & & 0.16 & 0.24 & $86 \pm 2$ & $2960 \pm 263$ & $68 \pm 1$ & $168 \pm 51$ \\
\hline & \multirow[t]{3}{*}{5.0} & 0.00 & 0.00 & $61.6 \pm 0.1$ & $10,814 \pm 299$ & $57.7 \pm 0.1$ & $6909 \pm 159$ \\
\hline & & 0.08 & 0.12 & $78 \pm 1$ & $1321 \pm 281$ & $69.7 \pm 0.7$ & $2811 \pm 25$ \\
\hline & & 0.16 & 0.24 & - & - & - & - \\
\hline & \multirow[t]{3}{*}{7.4} & 0.00 & 0.00 & $60.8 \pm 0.1$ & $6957 \pm 207$ & $57.0 \pm 0.1$ & $11,026 \pm 1004$ \\
\hline & & 0.08 & 0.12 & $76 \pm 2$ & $2766 \pm 43$ & $69 \pm 1$ & $2423 \pm 465$ \\
\hline & & 0.16 & 0.24 & - & - & - & - \\
\hline
\end{tabular}


density of the DMPC bilayer surface at higher $\mathrm{pH}$ values due to anionic diclofenac incorporation, which may cause the formation of thicker hydration layers to avoid electrostatic repulsion.

\section{Discussion}

The interactions of diclofenac with phosphatidylcholine bilayers were characterized herein at $\mathrm{pH} 3.0,5.0$, and 7.4 to eventually distinguish the effects of each ionization state on the protective phospholipid layers of the gastric mucosa. Two main parameters were characterized: the drug's affinity for DMPC bilayers and the drug's effects on their structural properties. Concerning the former parameter, the protonated/ neutral form of the drug (neutral diclofenac) was found to have higher affinity for DMPC bilayers than the deprotonated/ ionized form of the drug (anionic diclofenac). Indeed, a similar $\mathrm{pH}$-dependent trend was previously reported for diclofenac in egg yolk phosphatidylcholine (Ferreira et al., 2005), but no further studies concerning neutral diclofenac were performed so far. These studies are of utmost importance because of the $\mathrm{pH}$ gradient found in the gastric mucosa (Bahari et al., 1982) and because of the variation of the $p K_{a}$ of acidic drugs in phosphatidylcholine bilayers. In fact, the $p K_{a}$ of diclofenac in DMPC bilayers $\left(p K_{m}=4.7 \pm 0.3\right.$ ) was found herein to increase almost 1 unit in comparison with the same constant in the aqueous phase $\left(p K_{a}=3.97 \pm 0.04\right)$ (Ferreira et al., 2005). This result is in line with the data reported for other acidic NSAIDs (de Castro et al., 2001; Pereira-Leite et al., 2018c) and confirms that neutral diclofenac may actually circulate in the gastric mucosa after oral administration. This evidence ultimately shows the relevance of clarifying the effect of neutral diclofenac on the protective phospholipid layers of the gastric mucosa to comprehensively understand the toxicity mechanisms of this pharmaceutical.

Beyond displaying higher affinity for DMPC bilayers, neutral diclofenac caused remarkable alterations in the structural properties of the DMPC bilayer. From gel to fluid phases, neutral diclofenac induced 1) an increase of the bilayer thickness and the straightening of the DMPC molecules in the $\mathrm{P}_{\beta}$, phase; 2) a reduction of the temperature and the cooperativity of the DMPC's main phase transition, probably by weakening the lipid-lipid interactions in the gel phases and by decreasing the lipid tilt angle; and 3) a decrease of the bilayer fluidity and an increase of the bilayer thickness in the fluid phase, probably by acting as a steric constraint for the dynamics of lipid molecules and for the trans-gauche isomerizations in the DMPC's acyl chains.

It is noteworthy that the neutral diclofenac-induced effects on the phosphatidylcholine bilayers were often more pronounced and/or distinct than that caused by anionic diclofenac. In fact, the diclofenac-induced decrease of the temperature and cooperativity of the main phase transition of DMPC bilayers at $\mathrm{pH} 7.4$ and 5.0 was previously reported elsewhere (Manrique-Moreno et al., 2009; Fernandes et al., 2018). However, more pronounced alterations were observed with neutral diclofenac in this study, considering equivalent membrane concentrations. Moreover, anionic diclofenac was reported to reduce the membrane fluidity of the egg yolk phosphatidylcholine bilayer region probed by ( \pm )-2-(9-anthroyloxy)stearic acid (2-AS) but not by 6 -AS, 9 -AS, or 12 -AS (Ferreira et al., 2005). In contrast, neutral diclofenac was found in this work to decrease the bilayer fluidity of the inner region of the DMPC bilayer probed by DPH. This result is in line with the ordering effect recently reported for neutral acemetacin and indomethacin in the fluid phase of DMPC bilayers, using methyl 5-doxyl-stearate as the embedded probe (Pereira-Leite et al., 2018b,c).

The differential effects observed and reported for neutral diclofenac and anionic diclofenac may result from the distinct preferential location of each ionization state within the DMPC bilayer. Experimental data from this study suggested that neutral diclofenac has a deeper preferential location in the DMPC bilayer, putatively establishing H-bonds with the DMPC's phosphate group and disrupting the lipid-lipid interactions in the inner region of the acyl chains. On the other hand, anionic diclofenac seems to have a shallower location, probably because of electrostatic adsorption in the DMPC's choline group, as previously reported (Ferreira et al., 2005; Manrique-Moreno et al., 2009; Fernandes et al., 2018). This hypothesis is in agreement with molecular dynamics simulations that indicate that neutral NSAIDs are located closer to the bilayer center than anionic NSAIDs (Boggara and Krishnamoorti, 2010; Markiewicz and Pasenkiewicz-Gierula, 2011).

This study constitutes a key step in the attempt to fully describe the toxicity mechanisms of diclofenac in the gastric mucosa. Beyond the systemic actions of NSAIDs concerning the inhibition of protective PG biosynthesis, NSAID-induced topical actions also seem to be crucial to the occurrence of gastric adverse events. Anionic diclofenac may be harmful by ion trapping in the epithelial cells, as well as by modifying the structural properties of cell and organelle membranes, as previously suggested (Ferreira et al., 2005; Fernandes et al., 2018). By modifying organelle membranes, anionic diclofenac may indirectly inhibit COX isoenzymes (monotopic membrane proteins), causing an additional reduction of protective PGs, which may amplify the gastric toxicity. Additionally, neutral diclofenac seems to be able to pronouncedly alter the structural properties of the external phospholipid layers of the gastric mucosa. By modifying these protective barriers, neutral diclofenac may facilitate the penetration of toxic agents, such as protons and digestive enzymes, in the inner layers of the gastric mucosa, triggering pathophysiologic processes responsible for potential life-threatening adverse events.

It is conceivable that the aforementioned toxicity mechanism is shared by all acidic and nonselective NSAIDs. Indeed, it was already demonstrated that acemetacin also exerts distinct alterations in phospholipid bilayers according to the ionization state of the drug (Pereira-Leite et al., 2018c), in line with the data showed herein for diclofenac. Moreover, the anionic and neutral forms of ibuprofen were also found to establish differential interactions with phosphatidylcholine bilayers (Boggara et al., 2012), in agreement with the proposed hypothesis. Because of the chemical heterogeneity of NSAIDs, more studies are needed to ultimately confirm that the alteration of phospholipid bilayers by both neutral and acidic forms is a key toxicity mechanism of all acidic and nonselective NSAIDs.

In this sense, the toxic actions of both ionization forms of the drug must be considered in the development of new strategies to improve gastric tolerability. Indeed, two main approaches have been followed so far to improve the gastric safety of NSAIDs: 1) the design of new and/or hybrid compounds to 
treat inflammation (Pereira-Leite et al., 2017) and 2) the development of drug delivery systems to avoid gastric release (Pereira-Leite et al., 2018a). Among them, only one strategy has already reached the US market: the PLxGuard delivery system from PLx Pharma Inc. (Houston, TX), which uses phospholipids and free fatty acids to selectively release the NSAIDs in the duodenum (https://plxpharma.com/productstech/). Notably, this strategy was first developed by preassociating the drug with the phospholipids to ultimately hamper their in vivo association and the NSAID-induced alterations in the protective phospholipid layers of the gastric mucosa (Lichtenberger et al., 1995). Therefore, the potential application of studying the NSAID-phospholipid interactions is huge, not only as a vital source of information about the drugs' mechanisms of toxicity but also as important starting points for conceiving better therapeutic strategies.

\section{Acknowledgments}

The authors thank Manuela Barros and Vânia Dias for administrative and technical support, Joana C. Ricardo and Aleksander Fedorov for support during acquisition of the time-resolved fluorescence anisotropy data, and Christina S. Kamma-Lorger for help and support during beamtime in CELLS-ALBA Synchrotron [Proposal ID 2016021579]. The authors also thank Fundação para a Ciência e a Tecnologia, Programa Operacional Capital Humano, and European Union [Grants: SFRH/BD/109621/2015, IF/00293/2015, UIDB/04565/ 2020, SAICTAC/0019/2015], Conselho Nacional de Desenvolvimento Científico e Tecnológico [Grants: 160446/2013-9, Proc. 301250/20138], Fundação de Amparo à Pesquisa do Estado de São Paulo [Grant: Projeto Temático 2013/08166-5], and INCT-FCx (Instituto Nacional de Ciência e Tecnologia de Fluidos Complexos).

\section{Authorship Contributions}

Participated in research design: Pereira-Leite, Nunes, Reis.

Conducted experiments: Pereira-Leite, Jamal, Almeida, Nunes, Reis. Performed data analysis: Pereira-Leite, Jamal, Almeida, Coutinho. Wrote or contributed to the writing of the manuscript: PereiraLeite, Coutinho, Prieto, Cuccovia, Nunes, Reis.

\section{References}

Akabori K and Nagle JF (2015) Structure of the DMPC lipid bilayer ripple phase. Soft Matter 11:918-926.

Altman R, Bosch B, Brune K, Patrignani P, and Young C (2015) Advances in NSAID development: evolution of diclofenac products using pharmaceutical technology. Drugs 75:859-877.

Avdeef A (2001) Physicochemical profiling (solubility, permeability and charge state). Curr Top Med Chem 1:277-351.

Bahari HM, Ross IN, and Turnberg LA (1982) Demonstration of a $\mathrm{pH}$ gradient across the mucus layer on the surface of human gastric mucosa in vitro. Gut 23:513-516. Boggara MB and Krishnamoorti R (2010) Partitioning of nonsteroidal antiinflammatory drugs in lipid membranes: a molecular dynamics simulation study. Biophys J 98:586-595.

Boggara MB, Mihailescu M, and Krishnamoorti R (2012) Structural association of nonsteroidal anti-inflammatory drugs with lipid membranes. J Am Chem Soc 134: 19669-19676.

Conaghan PG (2012) A turbulent decade for NSAIDs: update on current concepts of classification, epidemiology, comparative efficacy, and toxicity. Rheumatol Int 32: 1491-1502.

de Castro B, Gameiro P, Lima JLFC, Matos C, and Reis S (2001) A fast and reliable spectroscopic method for the determination of membrane--water partition coefficients of organic compounds. Lipids 36:89-96.

Eisenblätter J and Winter R (2006) Pressure effects on the structure and phase behavior of DMPC-gramicidin lipid bilayers: a synchrotron SAXS and 2H-NMR spectroscopy study. Biophys $J$ 90:956-966.

Fernandes E, Soares TB, Gonçalves H, Bernstorff S, Real Oliveira MECD, Lopes CM, and Lúcio M (2018) A molecular biophysical approach to diclofenac topical gastrointestinal damage. Int $J$ Mol Sci 19:E3411.

Ferreira H, Lúcio M, Lima JLFC, Matos C, and Reis S (2005) Effects of diclofenac on EPC liposome membrane properties. Anal Bioanal Chem 382:1256-1264.

Grancelli A, Morros A, Cabanas ME, Domenech O, Merino S, Vazquez JL, Montero MT, Vinas M, and Hernandez-Borrell J (2002) Interaction of 6-fluoroquinolones with dipalmitoylphosphatidylcholine monolayers and liposomes. Langmuir 18:9177-9182.

Hills BA and Lichtenberger LM (1985) Gastric mucosal barrier: hydrophobicity of stretched stomach lining. Am $J$ Physiol 248:G643-G647.
Jain MK and Wu NM (1977) Effect of small molecules on the dipalmitoyl lecithin liposomal bilayer: III. Phase transition in lipid bilayer. J Membr Biol 34:157-201. Jendrasiak GL and Estep TN (1977) The interaction of 1-anilino-8-naphthalenesulfonate with lipids: a nuclear magnetic resonance study. Chem Phys Lipids 18:181-198. Kaiser RD and London E (1998) Location of diphenylhexatriene (DPH) and its derivatives within membranes: comparison of different fluorescence quenching analyses of membrane depth. Biochemistry 37:8180-8190.

Kirchheiner J, Meineke I, Steinbach N, Meisel C, Roots I, and Brockmöller J (2003) Pharmacokinetics of diclofenac and inhibition of cyclooxygenases 1 and 2 : no relationship to the CYP2C9 genetic polymorphism in humans. $\mathrm{Br}$ J Clin Pharmacol 55:51-61.

Koynova R and Caffrey M (1998) Phases and phase transitions of the phosphatidylcholines. Biochim Biophys Acta 1376:91-145.

Kucerka N, Kiselev MA, and Balgavý P (2004) Determination of bilayer thickness and lipid surface area in unilamellar dimyristoylphosphatidylcholine vesicles from smallangle neutron scattering curves: a comparison of evaluation methods. Eur Biophys $J$ 33:328-334.

Lakowicz JR (2006) Principles of Fluorescence Spectroscopy, Springer US, New York. Lichtenberger LM, Graziani LA, Dial EJ, Butler BD, and Hills BA (1983) Role of surface-active phospholipids in gastric cytoprotection. Science 219:1327-1329.

Lichtenberger LM, Wang ZM, Romero JJ, Ulloa C, Perez JC, Giraud MN, and Barreto JC (1995) Non-steroidal anti-inflammatory drugs (NSAIDs) associate with zwitterionic phospholipids: insight into the mechanism and reversal of NSAID-induced gastrointestinal injury. Nat Med 1:154-158.

Lichtenberger LM, Zhou Y, Jayaraman V, Doyen JR, O'Neil RG, Dial EJ, Volk DE, Gorenstein DG, Boggara MB, and Krishnamoorti R (2012) Insight into NSAIDinduced membrane alterations, pathogenesis and therapeutics: characterization of interaction of NSAIDs with phosphatidylcholine. Biochim Biophys Acta 1821: 994-1002.

Magalhães LM, Nunes C, Lúcio M, Segundo MA, Reis S, and Lima JLFC (2010) Highthroughput microplate assay for the determination of drug partition coefficients. Nat Protoc 5:1823-1830.

Manrique-Moreno M, Garidel P, Suwalsky M, Howe J, and Brandenburg K (2009) The membrane-activity of Ibuprofen, Diclofenac, and Naproxen: a physico-chemical study with lecithin phospholipids [published correction appears in Biochim Biophys Acta (2011) 1808:1946]. Biochim Biophys Acta 1788:1296-1303.

Markiewicz M and Pasenkiewicz-Gierula M (2011) Comparative model studies of gastric toxicity of nonsteroidal anti-inflammatory drugs. Langmuir 27:6950-6961.

McGettigan P and Henry D (2013) Use of non-steroidal anti-inflammatory drugs that elevate cardiovascular risk: an examination of sales and essential medicines lists in low-, middle-, and high-income countries. PLoS Med 10:e1001388.

Nardone G, Laccetti P, Civiletti C, and Budillon G (1993) Phospholipid composition of human gastric mucosa: a study of endoscopic biopsy specimens. Gut 34:456-460.

Nunes C, Brezesinski G, Lopes D, Lima JLFC, Reis S, and Lúcio M (2011) Lipid-drug interaction: biophysical effects of tolmetin on membrane mimetic systems of different dimensionality. J Phys Chem B 115:12615-12623.

Nunes C, Lopes D, Pinheiro M, Pereira-Leite C, and Reis S (2013) In vitro assessment of NSAIDs-membrane interactions: significance for pharmacological actions. Pharm Res 30:2097-2107.

Pereira-Leite C, Carneiro C, Soares JX, Afonso C, Nunes C, Lúcio M, and Reis S (2013a) Biophysical characterization of the drug-membrane interactions: the case of propranolol and acebutolol. Eur J Pharm Biopharm 84:183-191.

Pereira-Leite C, Lopes-de-Araújo J, Cuccovia IM, Reis S, and Nunes C (2018a) Nanodelivery systems for NSAIDs: challenges and breakthroughs, in Nanoparticles in Life Sciences and Biomedicine (Neves AR and Reis S eds) pp 345-373, Pan Stanford Publishing, Singapore.

Pereira-Leite C, Nunes C, Bozelli JC Jr., Schreier S, Kamma-Lorger CS, Cuccovia IM and Reis S (2018b) Can NO-indomethacin counteract the topical gastric toxicity induced by indomethacin interactions with phospholipid bilayers? Colloids Surf B Biointerfaces 169:375-383.

Pereira-Leite C, Nunes C, Grahl D, Bozelli JC, Schreier S, Kamma-Lorger CS, Cuccovia IM, and Reis S (2018c) Acemetacin-phosphatidylcholine interactions are determined by the drug ionization state. Phys Chem Chem Phys 20:14398-14409.

Pereira-Leite C, Nunes C, Jamal SK, Cuccovia IM, and Reis S (2017) Nonsteroidal anti-inflammatory therapy: a journey toward safety. Med Res Rev 37:802-859.

Pereira-Leite C, Nunes C, and Reis S (2013b) Interaction of nonsteroidal antiinflammatory drugs with membranes: in vitro assessment and relevance for their biological actions. Prog Lipid Res 52:571-584

Petrache HI, Tristram-Nagle S, and Nagle JF (1998) Fluid phase structure of EPC and DMPC bilayers. Chem Phys Lipids 95:83-94.

Simonian HP, Vo L, Doma S, Fisher RS, and Parkman HP (2005) Regional postprandial differences in $\mathrm{pH}$ within the stomach and gastroesophageal junction. Dig Dis Sci 50:2276-2285.

Suwalsky M, Manrique M, Villena F, and Sotomayor CP (2009) Structural effects in vitro of the anti-inflammatory drug diclofenac on human erythrocytes and molecular models of cell membranes. Biophys Chem 141:34-40.

Sweetman S (2011) Martindale: The Complete Drug Reference, 37th ed, Pharmaceutical Press, London.

van Meer G, Voelker DR, and Feigenson GW (2008) Membrane lipids: where they are and how they behave. Nat Rev Mol Cell Biol 9:112-124.

Venable RM, Brown FLH, and Pastor RW (2015) Mechanical properties of lipid bilayers from molecular dynamics simulation. Chem Phys Lipids 192:60-74.

Wallace JL (2008) Prostaglandins, NSAIDs, and gastric mucosal protection: why doesn't the stomach digest itself? Physiol Rev 88:1547-1565.

Address correspondence to: Catarina Pereira-Leite, Laboratório de Química Aplicada, Departamento de Ciências Químicas, Faculdade de Farmácia, Universidade do Porto, Rua de Jorge Viterbo Ferreira, 228, 4050-313 Porto, Portugal. E-mail: mleite@ff.up.pt 